\title{
A Theorem Relative to the Centrifugal Force Exerted Over a Relativistic Quantum Particle
}

\author{
M.A. Grado-Caffaro and M. Grado-Caffaro*
}

C/ Julio Palacios 11, 9-B, 28029-Madrid, Spain

\begin{abstract}
We prove that a free relativistic rotor (or rotator) Klein-Gordon field is massless. To this end, the magnitude of the centrifugal force and its associated quantum operator for a free particle with a circular motion are determined in Special Relativity. A partial differential equation including the centrifugal force operator is derived. Finally, from the conjunction of the above equation and the Klein-Gordon equation, the desired result is reached.
\end{abstract}

Keywords: Centrifugal force, special relativity, centrifugal force operator, massless rotor Klein-Gordon field.

\section{INTRODUCTION}

Issues related to the mass of quantum particles are certainly very interesting $[1,2]$. Consider, for example, the neutrino rest-mass. In this respect, the Standard Model predicts a zero rest-mass for the neutrino but, in this case, the mass (although very small) is non-zero. One may speak of massless Dirac or Klein-Gordon fields as idealization of more realistic models. In other words, particles with exactly zero rest-mass do not exist (contrary to pseudo-particles or quasi-particles, which, of course, have zero mass) so models based upon massless particles in relation to scalar or vector quantum fields are only approximations although these approximations are manifestly fruitful. Furthermore, an exciting problem arises: elucidating the mechanisms by which a given particle should acquire mass. At this point, one may think on a given quantum field which can impart mass to some class of particles.

The above considerations constitute one of the two basic ingredients of the present paper. The other ingredient refers to the centrifugal force exerted over a quantum-mechanical particle with circular motion in the framework of Special Relativity. As a matter of fact, we refer to a free rotor KleinGordon field which will be found to be massless. This is the main result of the paper. To achieve this, we will employ the concept of relativistic centrifugal force and its associated quantum operator. We refer to Special Relativity although centrifugal force within General Relativity plays also a significant role [3]. At any rate, the force in question has notorious relevance in Special Relativity by considering, for instance, hadron colliders. Certainly, acceleration of particles in the aforementioned devices has enormous importance. On the other side, since every solution of the Dirac equation is a solution of the Klein-Gordon equation, being the reciprocal assertion not true in general, all the fermions (except spinless fermions) cannot be included in our framework; consider, for

*Address correspondence to this author at the C/ Julio Palacios 11, 9-B, 28029-Madrid, Spain;

E-mail:ma.grado-caffaro@sapienzastudies.com example, electrons rotating under a perpendicular magnetic field. In this case, it is well-known that the magnitude of the centripetal force represented by the Lorentz force equals the magnitude of the centrifugal force. On the other hand, Landau states occupied by the electrons satisfying the KleinGordon equation should be regarded if we ignore the electron spin (see, for example, ref. [4]). Nevertheless, if we take into account the electron spin, electrons must not be regarded here because we restrict ourselves to spinless particles as, for instance, pions (the Klein-Gordon equation is essentially valid for spinless particles). In addition, the electron rest-mass is considerable so it cannot be viewed as quasi-zero in mathematical-physics models. We emphasize that we will consider particles obeying the Klein-Gordon equation but not obeying the Dirac equation. However, as an exception, the neutrino will be included in our framework. At this point, we note that a typical particle with very small rest-mass (which, as we have said above, was predicted to be zero by the Standard Model) is the neutrino.

\section{THEORY}

As really a fictitious force, the centrifugal force on a quantum, field-free, particle with circular motion has a magnitude which, in Special Relativity, reads:

$$
F=\frac{2 c}{r}\left(\sqrt{p^{2}+m_{0}^{2} c^{2}}-m_{0} c\right)
$$

where $r$ is the radius of the circumferential trajectory, $p$ is the magnitude of the momentum of the particle, $m_{0}$ denotes its rest-mass, and $c$ is the speed of light in vacuum.

The magnitude of the centrifugal force can also be expressed as:

$F=\frac{2 m_{0} c^{2}}{r}\left(\frac{1}{\sqrt{1-\frac{v^{2}}{c^{2}}}}-1\right)$

where, of course, $v$ is the magnitude of the particle velocity. 
At this point, consider the well-known formula namely $p=m_{0} v / \sqrt{1-c^{-2} v^{2}}$. Of course, inserting this last relation into (1), one finds (2). On the other hand, by expanding (2) in Taylor series around $v=0$ up to the second order, we get $F \approx m_{0} v^{2} / r$ which represents the non-relativistic case, as expected.

Now we enunciate:

Theorem.- A free relativistic rotor Klein-Gordon field (particles in circular motion) is massless.

Proof: We will prove the above result by using the concept of relativistic centrifugal force whose magnitude is given in formulas (1) or (2). Then, we determine the quantum operator for (1) so we have, given that $\hat{p} \equiv-i \hbar \nabla$ :

$\hat{F} \equiv \frac{2 c}{r}\left(\sqrt{m_{0}^{2} c^{2}-\hbar^{2} \nabla^{2}}-m_{0} c\right)$

On the other side, the quantity which multiplies to $2 / r$ in eq.(1) is the total energy of the particle so its associated Hamiltonian operator acting on the corresponding Hilbert space of wavefunctions is:

$\hat{H} \equiv c \sqrt{m_{0}^{2} c^{2}-\hbar^{2} \nabla^{2}}$

Therefore, from the conjunction of formulas (3) and (4), it follows:

$\hat{H} \equiv m_{0} c^{2}+\frac{r}{2} \hat{F}$

Next we will consider the well-known time-dependent Schrödinger equation:

$\hat{H}|\psi\rangle=i \hbar \frac{\partial}{\partial t}|\psi\rangle$

Applying $\hat{H}$ at both sides of eq.(6), it follows:

$\hat{H}^{2}|\psi\rangle=-\hbar^{2} \frac{\partial^{2}}{\partial t^{2}}|\psi\rangle$

Applying $\hat{H}$ at both sides of (5) and equating the resulting expression to the right-hand side of (7), one gets:

$\hat{F}^{2}|\psi\rangle+\frac{2 m_{0} c^{2}}{r} \hat{F}|\psi\rangle=-\frac{4 \hbar^{2}}{r^{2}} \frac{\partial^{2}}{\partial t^{2}}|\psi\rangle$

Now we regard the relativistic Klein-Gordon equation namely:

$\nabla^{2}|\psi\rangle-\frac{m_{0}^{2} c^{2}}{\hbar^{2}}|\psi\rangle=\frac{1}{c^{2}} \frac{\partial^{2}}{\partial t^{2}}|\psi\rangle$

which, since $\nabla \equiv(1 / r)(\partial / \partial \theta) \vec{u}_{\theta}$ with $\psi(\theta, t)$ where $\theta$ stands for the corresponding angular coordinate and $\vec{u}_{\theta}$ is the unit vector in the $\theta$-direction, then becomes:

$\frac{\partial^{2}}{\partial \theta^{2}}|\psi\rangle-\frac{m_{0}^{2} c^{2} r^{2}}{\hbar^{2}}|\psi\rangle=\frac{r^{2}}{c^{2}} \frac{\partial^{2}}{\partial t^{2}}|\psi\rangle$
By eliminating the second partial derivative of $|\psi\rangle$ with respect to time between eqs.(8) and (10), we have:

$\left(\frac{r^{2}}{4 c^{2}} \hat{F}^{2}+\frac{r m_{0}}{2} \hat{F}+\frac{\hbar^{2}}{r^{2}} \frac{\partial^{2}}{\partial \theta^{2}}\right)|\psi\rangle=m_{0}^{2}|\psi\rangle$

such that $\hat{M}_{0}^{2}|\psi\rangle=m_{0}^{2}|\psi\rangle$.

We must express the third term on the left-hand side of (11) in terms of $\hat{F}$ so that, from eq.(3) one gets:

$-\frac{\hbar^{2}}{r^{2}} \frac{\partial^{2}}{\partial \theta^{2}} \equiv \frac{r^{2}}{4 c^{2}} \hat{F}^{2}+\frac{r m_{0}}{2} \hat{F}$

Substituting (12) into (11), it follows that $\hat{M}_{0} \equiv \hat{0}$ so $m_{0}=0 \bullet$

Now we have:

Corollary 1.- Given that the relativistic-mass operator is $\hat{M} \equiv \hat{H} / c^{2}$, from the result above and relationship (4), one has that $\hat{M} \equiv-\frac{i \hbar}{c r} \frac{\partial}{\partial \theta}$.

Corollary 2.- Combining the result of the Theorem with formulae (4) and (5), it follows that $\hat{F} \equiv-\frac{2 i \hbar c}{r^{2}} \frac{\partial}{\partial \theta}$.

\section{CONCLUSIONS}

Starting from relationship (1), we have proved an interesting theorem after which the particles, with circular motion, obeying the Klein-Gordon equation but not obeying the Dirac equation, are massless which, in practice, means small enough rest-mass (consider pions and photons). In this respect, we emphasize that, for a particle obeying the Dirac equation, obeying the Klein-Gordon equation is a necessary (not sufficient) condition. In addition, notice that a particle of sufficiently small rest-mass may move with velocities extremely close to the speed of light in vacuum. Within this context, linear operators $\hat{F}, \hat{M}_{0}$ and $\hat{M}$ have been introduced. It is clear that, since $\hat{H}$ is self-adjoint, then both $\hat{F}$ and $\hat{M}$ are also self-adjoint (see formula (5) and Corollaries 1 and 2 ; notice that $\partial / \partial \theta$ is self-adjoint). On the other hand, operator $\hat{M}_{0}$ has found to be trivially self-adjoint (note that this operator is identically null). Finally, we wish to emphasize the relevance of our preceding study in the context of the theory of dynamical systems [5] and in relation to hadron colliders as well as we wish to remark that indirectly related work as ref. [6], which is certainly wrong, should be ignored.

\section{CONFLICT OF INTEREST}

The authors confirm that this article content has no conflict of interest.

\section{ACKNOWLEDGEMENTS}

Declared none. 


\section{REFERENCES}

[1] Winterberg F. The Planck aether hypothesis: an attempt for a finitistic theory of elementary particles. Germany: VRI, Karlsbad 2000.

[2] Winterberg F. Planck mass rotons as cold dark matter and quintessence. Z Naturforsch A 2002; 57: 202-4.
[3] Jonsson R. An intuitive approach to inertial forces and the centrifugal force paradox in general relativity. Am J Phys 2006; 74: 905-16.

[4] Skjervold JE, Ostgaard E. Matter in superstrong magnetic fields. Can J Phys 1986; 64: 256-68.

[5] Nitecki Z. Differentiable dynamics. Massachusetts, Cambridge: MIT Press 1971.

[6] Toharia M, Trodden M. Metastable kinks in the orbifold. Phys Rev Lett 2008; 100: 041602-5.

Received: September 19, 2012

Revised: September 26, 2012

Accepted: October 9, 2012

(C) Grado-Caffaro and Grado-Caffaro; Licensee Bentham Open.

This is an open access article licensed under the terms of the Creative Commons Attribution Non-Commercial License (http://creativecommons.org/licenses/bync/3.0/) which permits unrestricted, non-commercial use, distribution and reproduction in any medium, provided the work is properly cited. 